\title{
EDITORIAL
}

\section{Safety from farm to fork}

\author{
June marks Food Safety Week in the United Kingdom. Several recent lethal outbreaks remind \\ us that food-borne infections remain an important public health problem.
}

Earlier this year, peanuts contaminated with Salmonella killed nine people in the United States and led to the hospitalization of hundreds. In March, the results of the public inquiry into the second largest outbreak of Escherichia coli $\mathrm{O} 157$ in the United Kingdom, and the sixth largest outbreak worldwide, were made available. This outbreak, which occurred in Wales in 2005, caused more than 100 cases of illness and led to 1 death.

Such outbreaks show that food safety is not something that can be taken for granted in spite of the progress that has been made in recent years with the introduction of stricter regulations, improved food handling in the home as a result of awareness campaigns, a better understanding of the microbiology of food contamination and improved surveillance. The number of food poisoning cases remains high. In the United States alone, an estimated 70 million people suffer from food poisoning each year (although many cases are so mild that people do not seek treatment), leading to 300,000 hospitalizations and 5,000 deaths. In the United Kingdom, a similar percentage of the population is affected: in 2000, more than 400 deaths and over 81,000 cases of food poisoning by the most common foodborne pathogens, Salmonella spp., Campylobacter spp., E. coli O157, Listeria monocytogenes and Clostridium perfringens, were reported.

A new report released by the CDC in April showed that the incidence of food poisoning in the United States has reached a plateau after years of steady decreases. This is thought to be partly due to an increase in food poisoning cases related to contaminated fruits and vegetables, which has offset the decrease in cases that occurred as a result of food safety improvements in the meat and poultry industries. Another possibility is that all the easy food safety improvements have now been implemented, making additional gains both expensive and difficult.

However, there are reasons to be optimistic that further food safety improvements are possible. One is awareness campaigns such as Food Safety Week in the United Kingdom. Run by the Food Standards Agency, this campaign aims to educate the public on the dangers of food poisoning and provides information on how to decrease the risk of acquiring a food-borne infection. Spurred by the rapid increase in the number of
L. monocytogenes cases that disproportionally affect the elderly, the focus of the campaign this year is food safety for people aged over 60 . In recent years, listeriosis has killed more people in this age group than Salmonella and E. coli combined. The campaign provides information on ways to decrease exposure to contaminated food, how to store food correctly (such as checking the temperature in the refrigerator and following storage instructions on the labels) and how to handle food safely (for example, by not eating food past its 'use by' date), and also stresses the 4C's: cooking, cleaning, chilling and preventing cross-contamination. Greater awareness of food safety at home will undoubtedly lead to fewer cases of food poisoning and prevent unnecessary hospitalizations and deaths.

But consumers cannot remove all the risks by themselves, especially when it comes to processed and readyto-eat food. As the contaminated peanuts in the United States and E. coli outbreak in Wales show, there are holes in the food industry surveillance system. In both cases, the improper food handling that was the cause of the contamination was not dealt with effectively by inspectors. In part this was due to deceptive practices by the producer, but as food producers in the United States are not required to inform the public, or even the regulators, when they detect pathogens in their products, fundamental flaws in the production process can easily be concealed. Also, as the public inquiry on the Welsh outbreak showed, the infrastructure can make it difficult for inspectors to convey their concerns.

The publicity surrounding the recent outbreaks in the United States has led to calls for increased regulation of food producers by the Food and Drug Administration, which regulates approximately $80 \%$ of all US food production. Increased funding for additional inspectors to increase the frequency of inspections and increased openness on the side of the food producers are required to improve food safety. The report on the outbreak in Wales provides clear guidelines for improvements that can be made in the United Kingdom, which in part deal with stricter enforcement of the rules and clearer guidelines for reporting suspicions of violations.

Together, awareness campaigns and stronger, better-funded agencies might get us off the plateau and improve food safety further. Bon appétit! 